\title{
Zeitlich aufgelöste Modellierung des Energieverbrauchs bei der Elektrostahlproduktion
}

\author{
J. Dock (D, D. Janz, J. Weiss, A. Marschnig, M. Rahnama Mobarakeh, T. Kienberger
}

Die Stahlherstellung entlang der Elektrolichtbogenofenroute hat das Potenzial, einen wesentlichen Beitrag zur Dekarbonisierung der Stahlindustrie zu leisten. Das Erschmelzen von Stahlschrott und direkt reduziertem Eisen im Elektrolichtbogenofen (EAF) stellt eine Alternative zur energie- und emissionsintensiven integrierten Stahlproduktion mittels Hochofen und Konverter dar. Entscheidend für die Herstellung von $\mathrm{CO}_{2}$-armen Stahl im sogenannten Elektrostahlwerk ist jedoch die Steigerung der Energieeffizienz sowie der Einsatz erneuerbarer Energie im Produktionsprozess. Im Rahmen der vorliegenden Studie beschreiben wir die Entwicklung eines vollständigen, energieträgerübergreifenden, zeitlich und technologisch aufgelösten Energiesystemmodells eines Elektrostahlwerkes, welches der Bewertung von Energieeffizienzmaßnahmen sowie der Integration erneuerbarer Energie dient.

Schlüsselwörter: Energiesystemmodell; Lastprofil; Elektrolichtbogenofen; Stahlproduktion; Erneuerbare Energie

Time-resolved energy consumption modelling of electric steel production.

Steel production via the electric arc furnace route has the potential to contribute significantly to the decarbonization of the steel industry. The melting of steel scrap and direct reduced iron in the electric arc furnace (EAF) represents an alternative to the energy and emission-intensive integrated steel production based on blast furnace and converter. However, a crucial prerequisite for the production of low- $\mathrm{CO}_{2}$ steel in the so-called electric steel mill is the increase of energy efficiency as well as the utilization of renewable energy in the production process. Within the scope of this study, we describe the development of a holistic as well as a temporally and technologically resolved energy system model of an electric steel mill, which serves to evaluate energy efficiency measures and the integration of renewable energy.

Keywords: energy system model; load profile; electric arc furnace; steel production; renewable energy

Eingegangen am 13. April 2021, angenommen am 17. Mai 2021, online publiziert am 28. Mai 2021

(c) The Author(s) 2021

\section{Einführung}

Die österreichische Eisen- und Stahlindustrie bezog im Jahr 2017 insgesamt 36.7 TWh an Primärenergie [16] und emittierte 12.7 Mio. t Kohlenstoffdioxid [18]. Das entsprach $13.6 \%$ der Treibhausgasemissionen Österreichs. Verglichen mit der integrierten Prozessroute (Hochofen/Konverter) bietet die Elektrolichtbogenofenroute (Stahlschrott/EAF) die Möglichkeit, den Energieverbrauch und $\mathrm{CO}_{2}-$ Ausstoß in der Stahlproduktion zu reduzieren. Durch das Erschmelzen von Schrott im Elektrolichtbogenofen (EAF) entfällt der energieund emissionsintensive Reduktionsschritt. Im Jahr 2018 erfolgten in Europa $41 \%$ der Stahlproduktion über die EAF-Route (Eurofer 2019).

Betreibt man den Elektrolichtbogenofen mit elektrischer Energie aus erneuerbaren Quellen, lassen sich die spezifischen $\mathrm{CO}_{2}$ Emissionen weiter senken. Auch die Weiterverarbeitung von direktreduziertem Eisen (DRI) im Elektrolichtbogenofen führt gegenüber der Hochofenroute zu erheblichen Emissionseinsparungen [1]. Bhaskar et al. [3] beschreiben die Direktreduktion von Eisenerz mit Wasserstoff und die anschließende Stahlproduktion über die sogenannte HDRI-EAF-Route als klimafreundliche Alternative zur integrierten Stahlproduktion. Deren wirtschaftliche Umsetzbarkeit hängt lediglich von den Kosten für Elektrolyseanlagen und elektrische Energie ab. Voraussetzung für die Dekarbonisierung der Stahlproduktion ist jedoch die Verfügbarkeit von erneuerbarer elektrischer Energie [14].

\subsection{Produktionsprozess}

Der Stahlerzeugungsprozess in dem im Rahmen dieser Studie betrachteten Elektrostahlwerk lässt sich wie folgt beschreiben:

Am Beginn des Produktionsprozesses steht das Erschmelzen von Stahlschrott im Elektrolichtbogenofen. Der Eintrag der Schmelzwärme erfolgt dabei hauptsächlich über die Strahlungswärme des Lichtbogens, welcher sich zwischen den Elektroden des Ofens und dem Schrott ausbildet, elektrische Widerstandserwärmung im Schrott und durch exotherme Oxidationsreaktionen gewisser Schrottbestandteile [4]. Der elektrische Energiebedarf des EAF liegt laut Literatur zwischen 404 und 748 kWh/t Stahl. Beim sogenannten Frischen werden etwa 5 bis $65 \mathrm{~m}_{N}^{3}$ Sauerstoff pro Tonne flüssigen Stahls in die Schmelze eingeblasen [13]

Weitere Prozessschritte sind der Abstich des flüssigen Stahls in eine Stahlwerkspfanne sowie das Abschlacken. Als Sekundär- oder

Dock, Johannes, Lehrstuhl für Energieverbundtechnik, Montanuniversität Leoben, Parkstraße 31, A-8700 Leoben, Österreich (E-Mail: johannes.dock@unileoben.ac.at); Janz, Daniel, Breitenfeld Edelstahl AG, St. Barbara-Mitterdorf, Österreich; Weiss, Jakob, Lehrstuhl für Energieverbundtechnik, Montanuniversität Leoben, Leoben, Österreich; Marschnig, Aaron, Lehrstuhl für Energieverbundtechnik, Montanuniversität Leoben, Leoben, Österreich; Rahnama Mobarakeh, Maedeh, Lehrstuhl für Energieverbundtechnik, Montanuniversität Leoben, Leoben, Österreich; Kienberger, Thomas, Lehrstuhl für Energieverbundtechnik, Montanuniversität Leoben, Leoben, Österreich 


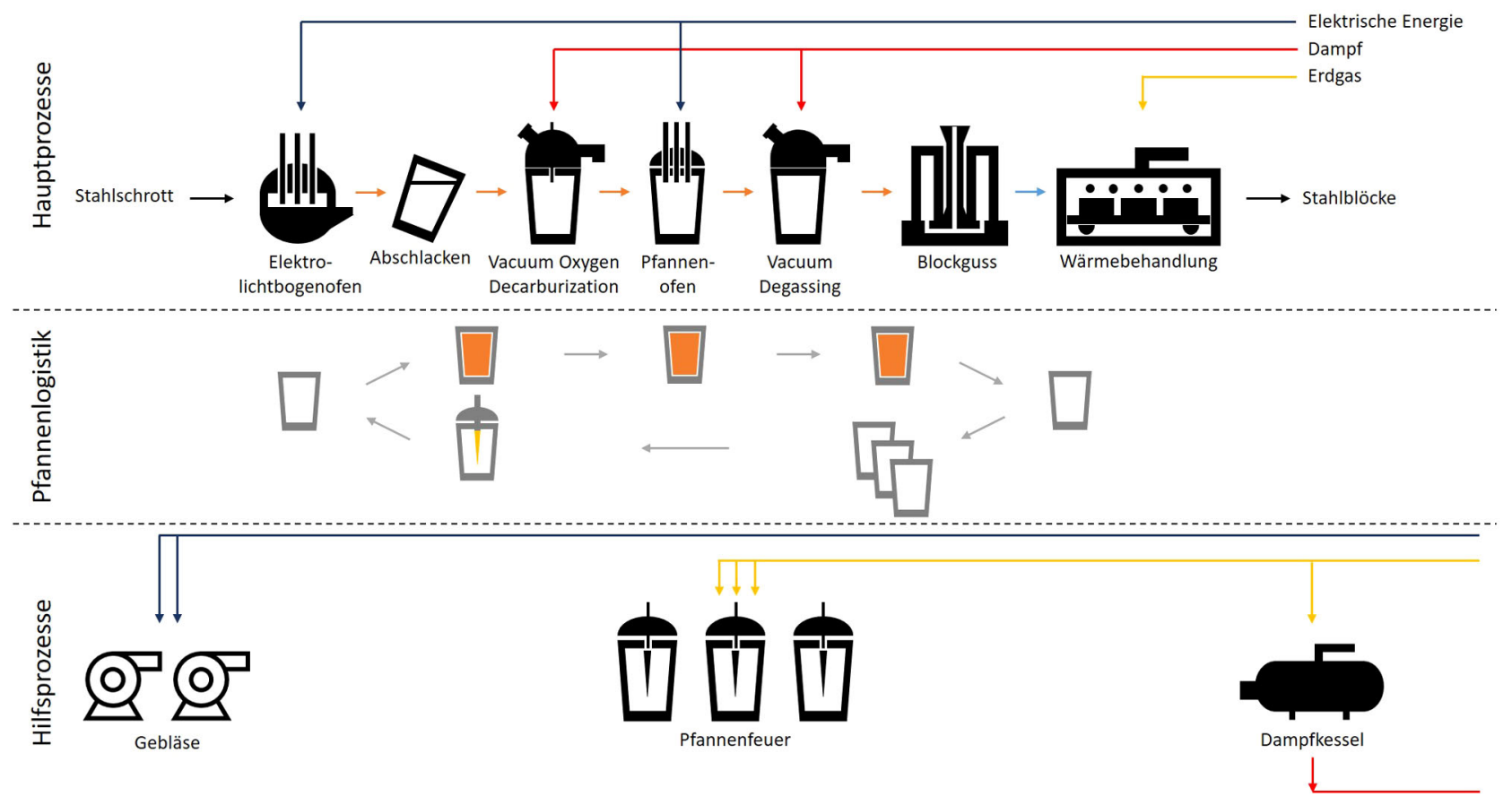

Abb. 1. Übersicht Energiesystemmodell

Pfannenmetallurgie kann man die folgenden Schritte zusammenfassen, welchen die Schmelze je nach gewünschter Qualität unterzogen wird: Entschwefelung, Desoxidation, Homogenisierung, Entgasung, Einstellung der chemischen Zusammensetzung sowie Entfernen von nichtmetallischen Einschlüssen [20].

Während der sekundärmetallurgischen Behandlungen wird die Temperatur der Schmelze mittels eines Pfannenofens eingestellt. Der Energieeintrag erfolgt dabei, wie beim EAF, über Elektroden.

Bei der Herstellung von Edelstählen kommt der Vacuum Oxygen Decarburization (VOD) sowie dem Vacuum Degassing (VD) besondere Bedeutung zu. Unter Vakuum werden dabei Kohlenstoff (VODVerfahren) und gelöste Gase (VD-Verfahren) aus dem flüssigen Stahl entfernt.

Hat die Schmelze die gewünschte Zusammensetzung und Temperatur erreicht, wird der Stahl in Kokillen vergossen. Die erstarrten Blöcke werden dann einer Wärmebehandlung unterzogen, um die Eigenschaften des Stahls einzustellen.

Energieintensive Hilfsprozesse im Stahlwerk sind die Entstaubung und das Vorheizen der Stahlwerkspfannen. Der Herstellungsprozess sowie die wesentlichen Energieverbraucher sind in Abb. 1 ersichtlich.

\subsection{Forschungsbedarf}

Mögliche Maßnahmen zur Dekarbonisierung der Elektrolichtbogenofenroute reichen von der Implementierung energieeffizienterer Sub-Prozesse bis hin zur Schaffung von Lastflexibilitäten zur verstärkten Integration erneuerbarer Energien. Für die Bewertung und Umsetzung solcher Maßnahmen bedarf es geeigneter Modelle, mit deren Hilfe sich die Auswirkungen von Änderungen im Produktionsprozess auf den Energieverbrauch, den CO2-Ausstoß sowie die Belastung vorgelagerter Netze abschätzen lassen.

In der Fachliteratur finden sich zahlreiche Studien, welche sich mit der Modellierung von Energiesystemen im Bereich der Stahlherstellung mittels EAF beschäftigen:
Thermodynamische Modelle bedienen sich des ersten bzw. zweiten Hauptsatzes der Thermodynamik, um Prozesse physikalisch korrekt abbilden zu können. Beispiele dafür sind das Energiemodell eines Elektrolichtbogenofens von Kirschen et al. [9] und jenes eines Elektrolichtbogen- bzw. eines Pfannenofens von Camdali und Tunc $[5,6]$.

Im Gegensatz zu thermodynamischen Modellen beruht die datengetriebene Modellierung von Energieverbräuchen auf der Analyse und Verknüpfung großer Datenmengen. Im Bereich der Energiemodellierung von Elektrolichtbogenöfen kommen neben anderen Methoden Regressionsmodelle, genetische Programmierung [10] und neuronale Netze zum Einsatz [7, 8].

Während es mithilfe dieser Modelle möglich ist, den Energieverbrauch einzelner Aggregate vorherzusagen und zu bewerten, können keinerlei Aussagen in Hinblick auf den zeitlichen Energieverbrauch oder auf die Wechselwirkung mit anderen Verbrauchern im Sinne einer Prozessintegration getroffen werden.

Ein Ansatz zur Beschreibung von Energiesystemen ist Agent-Based Modelling. Zarandi und Ahmadpour [20] schlagen in ihrer Studie einen solchen Ansatz zur Modellierung des Stahlwerksprozesses vor, bei dem die einzelnen Sub-Prozesse als eigenständige Agenten implementiert werden, die durch gegenseitige Interaktion den Gesamtprozess abbilden.

Prozessmodelle, wie jenes von Matino et al. [11], basieren auf der Nachbildung von Gesamtproduktionsprozessen. In dieser Arbeit wird ein virtuelles Stahlwerk zur Berechnung von Key Performance Indicators (KPIs) wie spezifische Energieverbräuche oder $\mathrm{CO}_{2}$-Emissionen herangezogen.

Sowohl das beschriebene agentenbasierten Modell als auch das Prozessmodell eignet sich für die energietechnische Bewertung gesamter Energiesysteme, jedoch geben auch sie keinen Aufschluss über die Lastprofile beziehungsweise die energietechnische Interaktion der einzelnen Komponenten oder des gesamten Energiesystems. 
Mathematische Modelle erlauben die energetische, ökonomische oder ökologische Optimierung von Energiesystemen. Atabay [2] präsentiert ein mathematisches Modell zur wirtschaftlichen Optimierung industrieller Energiesysteme. Dieses Modell berücksichtigt zeitliche Energieverbräuche, allerdings sind diese Lastprofile der Ausgangspunkt der Optimierungsrechnung und müssen folglich, etwa aus Messungen, bekannt sein.

Die Produktion von Stahl entlang der Elektrolichtbogenofenroute besteht aus einer Reihe von komplexen Batchprozessen. Daher ist der Energieverbrauch einzelner Aggregate sowie des gesamten Stahlwerkes starken Fluktuationen unterworfen. Um den Energieverbrauch dieser Prozesse adäquat beschreiben zu können, entwickeln wir in der vorliegenden Arbeit ein technologisch und zeitlich aufgelöstes Energiesystemmodell eines Elektrostahlwerkes. Dieses soll mit geringem Messaufwand sowohl synthetische Lastprofile einzelner Aggregate sowie des gesamten Energiesystems als auch energietechnische KPIs liefern und ermöglicht damit:

- die Untersuchung neuartiger Prozesslayouts, wie z.B. Energierückgewinnung durch die Implementierung von Speichern,

- die Bewertung von Möglichkeiten zur Betriebsoptimierung,

- die Integration volatiler erneuerbarer Energie in Produktionsprozesse, sowie

- die Bewertung der Auswirkungen von Änderungen im Prozessdesign auf vorgelagerte Energienetze.

Dieser Artikel ist folgendermaßen gegliedert: Der Darstellung des Produktionsprozesses (Abschn. 1.1) und des Forschungsbedarfs (Abschn. 1.2) folgt eine Beschreibung der Modellierungsmethodik (Abschn. 2). Abschnitt 3 umfasst die Präsentation sowie die Diskussion der Ergebnisse. Die wichtigsten Erkenntnisse sind in der Schlussfolgerung (Abschn. 4) zusammengefasst.

\section{Methodik}

Unser Ansatz besteht in der Modellierung der einzelnen SubProzesse als Module, die im Anschluss zu einem Energiesystemmodell zusammengesetzt werden. Durch das Aggregieren der einzelnen Energieverbräuche in jedem Zeitschritt erhalten wir dann die Gesamtlastprofile für elektrische Energie und Erdgas.

\subsection{Modellierung der Sub-Modelle}

Im Zuge einer Energieanalyse des Stahlwerkes der Breitenfeld Edelstahl AG wurden die folgenden Aggregate aufgrund ihres Energieverbrauchs bzw. ihrer Nennleistung als wesentliche Komponenten des Energiesystems identifiziert:

- Elektrolichtbogenofen,

- Pfannenöfen,

- Pfannenfeuer,

- Wärmebehandlungsöfen,

- Prozessdampfkessel,

- Entstaubungsgebläse.

Die aufgelisteten Komponenten sind in Summe für jeweils über $80 \%$ des Strom- und Erdgasverbrauchs verantwortlich. Die übrigen Verbraucher werden aufgrund ihrer hohen Anzahl und folglich hohem Messaufwand als Grundlast modelliert.

Über mehrere Wochen wurden mittels Power Analyzer (Artemes AM 15-PLOG) und vorhandener Turbinenradzähler in Verbindung mit einem Datenlogger (DataTaker DT-85) die Lastprofile der oben genannten Komponenten gemessen. Die Profile weisen eine zeitliche Auflösung von einer Minute auf. Für die elektrischen Verbraucher wurden zusätzlich zur Wirkleistung auch die Blind- und Scheinleistung aufgezeichnet.
Gemäß der Form ihrer Lastprofile sowie der gefundenen Zusammenhänge lassen sich die Aggregate wie folgt einteilen: SubProzesse mit stark fluktuierenden, von den Vorprozessen abhängigen bzw. vordefinierbaren Lastprofilen. Entsprechen dieser Einteilung unterscheiden sich die jeweils eingesetzten Modellierungsmethoden (siehe 2.1.1, 2.1.2 und 2.1.3).

Für die Entwicklung der Sub-Modelle sowie des Gesamtmodells wurde Python als Programmiersprache ausgewählt. Vorteile dieser Sprache sind ihre vielseitigen Anwendungsmöglichkeiten, die Verfügbarkeit umfassender Dokumentationen und Tutorials, sowie eine breite Palette an Bibliotheken. Zur Erstellung dieses Modells wurden vor allem die Bibliotheken Pandas, Numpy sowie Matplotblib verwendet.

\subsubsection{Sub-Prozesse mit stark fluktuierenden Lastprofilen} Die Lastprofile des Lichtbogenofens und des Pfannenofens zeichnen sich durch eine stark veränderliche Leistungsaufnahme aus. Daher werden diese im Modell mittels Markov-Ketten synthetisiert.

Markov-Ketten dienen der Vorhersage zukünftiger Zustände eines Systems auf Basis historischer Daten. In der Energietechnik werden diese häufig angewandt, um synthetische Last- oder Erzeugungsprofile zu modellieren. Beispiele dafür sind Verbrauchsprofile von Haushalten [12], Windgeschwindigkeits- und Erzeugungsprofile für Windkraftanlagen [15] oder Ladeprofile für Elektrofahrzeuge [19].

Physikalische Prozesse, darunter auch zeitliche Energieverbräuche, lassen sich oft als Abfolge diskreter Zustände beschreiben. Hängt der nächste Zustand des zu beschreibenden Systems ausschließlich von dessen aktuellem Status ab, so erfüllt der Prozess die sogenannte Markov-Eigenschaft. Die Wahrscheinlichkeit, dass das System innerhalb eines Zeitschrittes $(n)$ von seinem aktuellen Zustand $(i)$ in einen gewissen nachfolgenden Zustand (j) übergeht, wird als Übergangswahrscheinlichkeit $\left(p_{i j}\right)$ bezeichnet. Die einzelnen Wahrscheinlichkeiten werden in einer Übergangswahrscheinlichkeitsmatrix angeordnet (1) [17].

$$
P(n)=\left(\begin{array}{ccccc}
p_{00}(n) & p_{01}(n) & \ldots & p_{0 j}(n) & \cdots \\
p_{10}(n) & p_{11}(n) & \ldots & p_{1 j}(n) & \cdots \\
\vdots & \vdots & \vdots & \vdots & \vdots \\
p_{i 0}(n) & p_{i 1}(n) & \cdots & p_{i j}(n) & \cdots \\
\vdots & \vdots & \vdots & \vdots & \vdots
\end{array}\right)
$$

Die kumulierte Übergangswahrscheinlichkeitsmatrix ergibt sich durch Aufsummieren der Einzelwahrscheinlichkeiten für jede Zeile gemäß der folgenden GI. (2).

$$
p_{i j, \text { cum }}=\sum_{k=0}^{j} p_{i k}
$$

Geben wir nun einen Ausgangszustand in Form eines Startvektors vor und ziehen eine diskrete Zahl $z$ sodass $0 \leq z \leq 1$, erhalten wir durch den Vergleich der Zufallszahl mit den kumulierten Übergangswahrscheinlichkeiten den nächsten Zustand. Dieses Prinzip wenden wir bei der Lastprofilmodellierung an.

Zur Generierung der Übergangswahrscheinlichkeiten wird die Nennleistung des Ofens in Leistungsintervalle unterteilt, die in weiterer Folge die unterschiedlichen Zustände darstellen. Zählt man die Anzahl der Übergänge für alle möglichen Zustandsfolgen im gemessenen Lastprofil und normiert diese, erhält man eine Übergangswahrscheinlichkeitsmatrix. 
Um physikalisch sinnvolle Ergebnisse in Hinblick auf Energieverbrauch und Prozesszeiten zu erhalten, werden dem Modell Verteilungen verschiedener Prozessgrößen als Inputwerte übergeben. Diese Prozessgrößen sind das Chargengewicht, die sogenannte Tap-totap-Zeit, die Zeitdauer zwischen zwei Abstichen, sowie der spezifische elektrische Energieverbrauch. Aus diesen Verteilungen wird für jede Prozesscharge ein Wert gezogen und aus dem spezifischen Energieverbrauch und dem Chargengewicht ein Gesamtenergieverbrauch berechnet. Die errechnete Energiemenge wird dann über die vorgegebene Tap-to-tap-Zeit auf eine Markov-Kette umgelegt.

\subsubsection{Sub-Prozesse mit abhängigen Lastprofilen}

Gebläse weisen eine Abhängigkeit zu den im Stahlwerk stattfindenden Prozessen auf, was auch ihrer Regelungsstrategie entspricht. Die Entstaubungsgebläse der Werkshallen sind immer dann im Einsatz, wenn das Stahlwerk in Betrieb ist und stehen andernfalls still, beispielsweise am Wochenende. Grundsätzlich wird die Entstaubungsanlage gleichförmig in Teillast betrieben und schaltet lediglich während des Blockgusses auf Volllast um.

Die Gebläse des Elektrolichtbogenofens haben die Aufgabe, Abgase aus dem Ofengefäß und durch eine Haube über dem Ofen abzuziehen. Diese Gebläse sind während des Schmelzbetriebs im Einsatz und werden bei Energieeintrag in den Ofen auf Volllast, andernfalls in Teillast betrieben.

Entsprechend der obenstehenden Analyse wurden die einzelnen Anlagen ins das Energiesystemmodell integriert: Die EAF-Gebläse beziehen während des Schmelzbetriebs Leistung auf Teillast- und bei zusätzlicher elektrischer Leistungsaufnahme des Elektrolichtbogenofens auf Volllastniveau. Analog dazu nehmen die Hallenentstaubungsgebläse während der Betriebszeiten des Stahlwerkes Leistung auf Teillast- und für die Zeitdauer des Gussprozesses auf Volllastniveau auf.

\subsubsection{Sub-Prozesse mit vordefinierbaren Lastprofilen}

Die verbleibenden Prozesse, die Vakuumbehandlungen (VD und VOD), die Wärmebehandlungsöfen und die Pfannenfeuer haben vorgegebene Prozesszeiten und festgelegte Lastprofile, welche bei der Modellierung berücksichtigt werden müssen.

Die Generierung des Vakuums für das VD- und VOD-Verfahren erfolgt mittels Dampfstrahlpumpen, die von zwei erdgasbetriebenen Prozessdampfkesseln gespeist werden. Über die Prozessdauer wird Dampf mit konstantem Massenstrom und konstanten Dampfparametern aus dem Kessel entnommen. Somit ergibt sich die erforderliche thermische Leistung des Kessels während der Evakuierung des Behandlungsgefäßes gemäß Gl. (3) aus dem Massenstrom $\left(\dot{m}_{\text {Dampf }}\right)$ und der spezifischen Enthalpie $\left(h_{\text {Dampf }}\right)$ des Dampfes sowie dem Wirkungsgrad des Kessels ( $\left.\eta_{\text {Kessel }}\right)$.

$$
P_{\text {thermisch }}(t)=\frac{\dot{m}_{\text {Dampf }}(t) \cdot h_{\text {Dampf }}}{\eta_{\text {Kessel }}}
$$

Das Lastprofil der Wärmebehandlungsöfen ist durch einen vorgegebenen Temperaturverlauf und eine definierte Prozessdauer gekennzeichnet. Daher wird im Modell ein gemessenes Lastprofil hinterlegt, welches von den im Modell implementierten Öfen nachgefahren wird.

Stahlwerkspfannen werden als Transport- und Behandlungsgefäße für flüssigen Stahl eingesetzt. Davor müssen sie jedoch mit sogenannten Pfannenfeuern auf Betriebstemperatur aufgeheizt werden. Hat eine Pfanne ihre Lebensdauer überschritten, erhält sie eine neue feuerfeste Zustellung und muss nach einem vorgegebenen Temperaturverlauf getrocknet werden. Auch diese Aufgabe übernehmen die Pfannenfeuer. Daraus ergeben sich für Pfannenfeuer zwei verschiedene Lastprofile: Vorheizen und Trocknen.
Um Aussagen über den Einsatz und den zeitlichen Erdgasverbrauch der einzelnen Pfannenfeuer zu ermöglichen, ist es notwendig diesen Kreislauf aus Vorheizen, Einsatz, Zustellung und Trocknung der Pfannen, die sogenannte Pfannenlogistik, zu modellieren (siehe Abb. 1). Dazu wird eine gewisse Anzahl an Pfannen-Objekten im Modell implementiert und einer Vorheiz-Warteschlange hinzugefügt. Ist ein freies Pfannenfeuer verfügbar, wird diesem eine Pfanne aus der Warteschlange zugewiesen. Im Falle eines Abstichs am Elektrolichtbogenofen beendet eines der Pfannenfeuer den VorheizProzess und die Pfanne wird einer Produktionscharge zugewiesen. Nach dem Guss entscheidet die Zahl der durchlaufenen Produktionszyklen, ob die Pfanne erneut zur Vorheizwarteschlange hinzugefügt wird oder Arbeiten an der Feuerfestzustellung erforderlich sind. In diesem Fall wird die Pfanne analog zum Vorheizen einer Warteschlange hinzugefügt. Sind Zustell-Kapazitäten verfügbar, wird sie zugestellt und anschließend, falls ein Pfannenfeuer verfügbar ist, getrocknet.

Der Aufbau des Pfannenlogistikmodells lässt sich vereinfacht wie folgt beschreiben:

für jeden Zeitschritt:

für Pfannen in Liste Vorheizen:

wenn Abstich am Elektrolichtbogenofen:

hinzufügen zu Liste Betrieb

erhöhen der Zyklenzahl um eins

für Pfannen in Liste Betrieb:

wenn Blockguss:

wenn Zyklenzahl kleiner als oder gleich Lebensdauer: hinzufügen zu Liste Warten auf Vorheizen

wenn Zyklenzahl größer als Lebensdauer:

hinzufügen zu Liste Warten auf Wartung

für Pfannen in Liste Warten auf Vorheizen:

wenn Pfannenfeuer verfügbar:

hinzufügen zu Liste Vorheizen

für Pfannen in Liste Warten auf Wartung:

wenn Wartungsplatz verfügbar:

hinzufügen zu Liste Wartung

für Pfannen in Liste Wartung:

wenn Wartung abgeschlossen:

hinzufügen zu Liste Warten auf Vorheizen

nullsetzen der Zyklenzahl

\subsection{Gesamtmodell}

Ziel dieser Arbeit ist die Entwicklung eines vollständigen, zeitlich aufgelösten Energiesystemmodells des Elektrostahlwerkes unter Berücksichtigung der eingesetzten Endenergieträger. Zu diesem Zweck werden die Sub-Modelle, welche als individuelle Module aufgebaut sind, zu einem Gesamtmodell verschmolzen. Abbildung 1 gibt einen Überblick über das Gesamtmodell. Zur besseren Übersicht ist dieses in Hauptprozesse, Hilfsprozesse und Pfannenlogistik gegliedert.

Um die Sub-Prozesse miteinander zu verbinden, werden im Modell Produktionschargen initialisiert, mit Eigenschaften wie etwa dem Chargengewicht oder Tap-to-tap-Zeiten ausgestattet und im Sinne einer sequenziellen Logik nacheinander verschiedenen Öfen und Behandlungen zugewiesen. Der erste Prozessschritt ist das Erschmelzen und anschließende Frischen von Stahlschrott im Elektrolichtbogenofen. Dort wird ein der errechnete Energiebedarf über die vordefinierte Tap-to-tap-Zeit mittels einer Markov-Kette auf ein elektrisches Lastprofil umgelegt.

Anschließend wird die Charge einer Warteschlange für die sekundärmetallurgischen Behandlungen hinzugefügt und nach vorgegebenen Wartezeiten freien Pfannenöfen, VD- und VOD-Anlagen zugewiesen. Hat die Charge alle erforderlichen Behandlungen durch- 

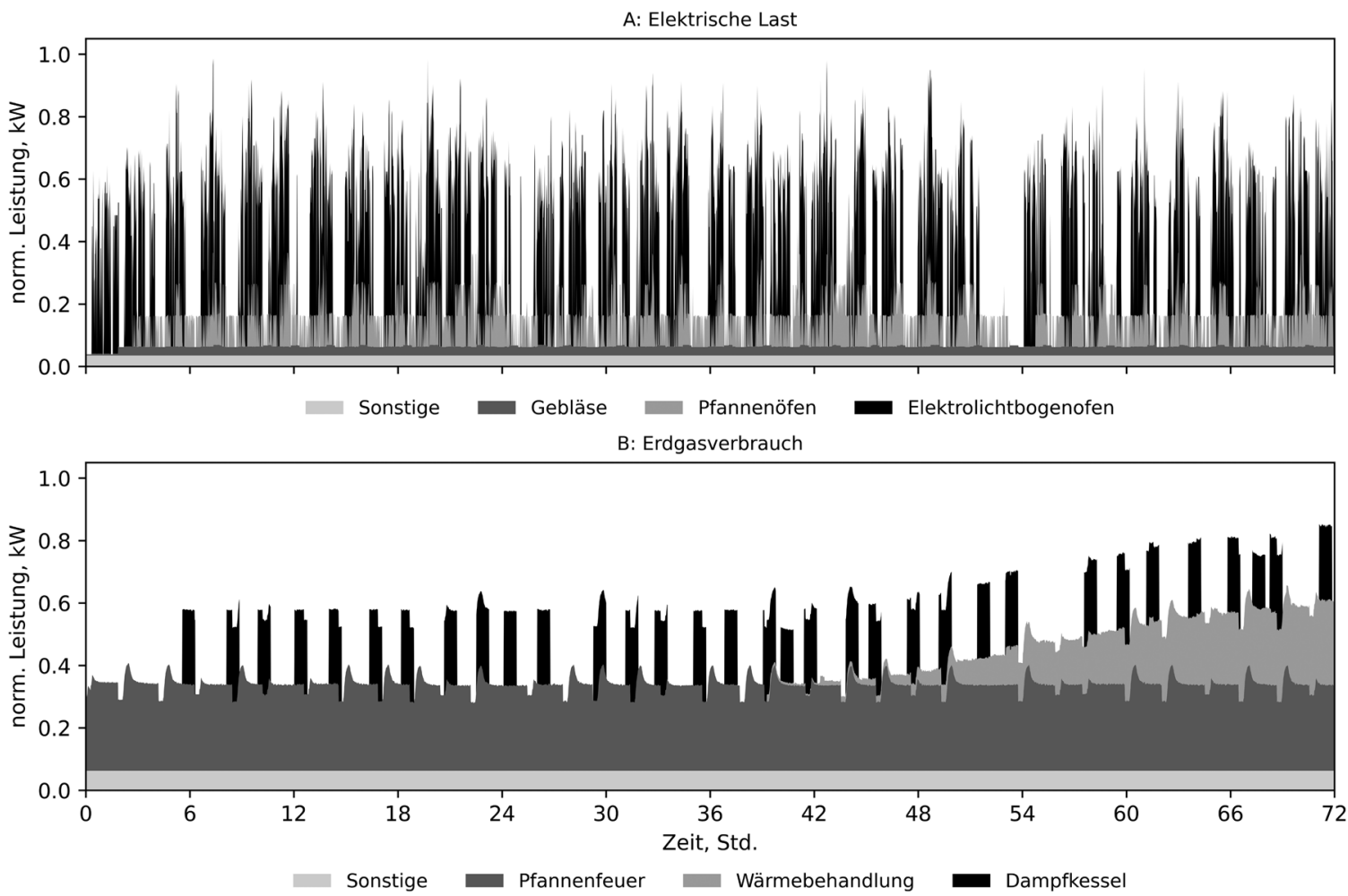

Abb. 2. Lastprofile für elektrische Energie $(A)$ und Erdgas $(B)$

laufen, erfolgt der Blockguss. Nach einer gewissen Auskühlzeit werden die Chargen einem Glühofen zur Wärmebehandlung übergeben. Übersteigt die Gesamtmasse der Chargen die Kapazität des Ofens, beginnt dieser mit dem Glühprogramm. Nach Beendigung der Wärmebehandlung gelten die Chargen als abgeschlossen.

Für jeden Zeitschritt iteriert das Modell durch die Liste an implementierten Aggregaten und ruft jeweils die aktuelle Leistungsaufnahme ab. Durch Aufsummieren der einzelnen Leistungen und der Grundlast entsteht ein aggregiertes Lastprofil des Stahlwerkes.

\section{Ergebnisse und Diskussion}

Eine Simulation mithilfe des Energiesystemmodells ergibt folgende auf die maximale Leistung normierte Gesamtlastprofile für elektrische Energie und Erdgas (siehe Abb. 2). Um die Validität der Lastprofile zu überprüfen, vergleichen wir die simulierten Energieverbräche der einzelnen Sub-Prozesse mit gemessenen Werten. Dazu stellen wir die spezifischen Energieverbräuche in kWh/t Stahl aus fünf Simulationsdurchläufen mit den spezifischen Energieverbräuchen der einzelnen Sub-Prozesse aus dem Jahr 2018 gegenüber und bilden den Mean Absolute Percentage Error (MAPE) (siehe Tab. 1).

Betrachtet man das Gesamtlastprofil, so ist der große Beitrag des Elektrolichtbogenofens zur elektrischen Last ersichtlich. Daher ist die Modellierung des EAF für die Erzeugung repräsentativer Lastprofile des Energiesystems entscheidend. Ähnlich verhält es sich mit dem Beitrag der Pfannenfeuer zum zeitlichen Erdgasbedarf. Aufgrund des vergleichsweise geringen Energieverbrauchs, der geringen installierten Einzelleistungen und des folglich relativ geringen Einflusses der übrigen Verbraucher auf die Gesamtlastprofile, ist deren Modellierung als Grundlast zulässig.

Besonders für die energie- und leistungsmäßig wesentlichen Verbraucher, wie etwa den EAF, die Wärmebehandlungsöfen oder die Pfannenfeuer liefert das in dieser Arbeit beschriebene Modell geringe Abweichungen von den gemessenen Jahresenergieverbräuchen.
Tab. 1. Abweichung des simulierten vom gemessenen spezifischen Energieverbrauch

\begin{tabular}{ll}
\hline Sub-Prozess & MAPE \\
\hline Elektrolichtbogenofen & $4.0 \%$ \\
Pfannenofen & $8.1 \%$ \\
Wärmebehandlungsöfen & $2.9 \%$ \\
Entstaubungsgebläse & $11.3 \%$ \\
Pfannenfeuer & $4.8 \%$ \\
Dampfkessel & $8.4 \%$ \\
Sonstige & $8.8 \%$ \\
Gesamt & $2.2 \%$ \\
\hline
\end{tabular}

Die Eignung des vollständigen Modells für energietechnische Berechnungen spiegelt sich in der geringen Gesamtabweichung des Energieverbrauchs von $2.2 \%$ wieder.

\section{Schlussfolgerungen}

Das in dieser Studie beschriebene Energiesystemmodell ist in der Lage, zeitlich aufgelöste Lastprofile der einzelnen Sub-Prozesse sowie des gesamten Stahlwerkes zu generieren.

Aufgrund des modularen Aufbaus des Modells ist es ohne Restrukturierung möglich, weitere Prozesse hinzuzufügen oder neue Technologien zu implementieren. Dadurch lassen sich Umstellungen im Produktionsprozess simulieren und deren Auswirkungen auf das Gesamtsystem bewerten.

Eine quantitative Evaluierung des simulierten spezifischen Energieverbrauchs des Gesamtsystems ergab eine Abweichung von unter $3 \%$.

Das vorliegende Energiesystemmodell wird in weiterer Folge um zeitlich aufgelöste Abwärme-, Sauerstoff- und Kohlendioxidströme 
ergänzt. Ziel ist die energietechnische Bewertung neuartiger Prozessdesigns unter Einbindung von Speichern zur Rückgewinnung und werksinternen Nutzung von Abwärme und $\mathrm{CO}_{2}$.

\section{Danksagung}

Diese Arbeit wurde im Rahmen des Projektes OxySteel durchgeführt. OxySteel ist ein Teilprojekt von NEFI - New Energie for Industry, eine vom österreichischen Klima- und Energiefonds finanzierte Vorzeigeregion Österreichs.

Funding Note Open access funding provided by Montanuniversität Leoben.

Hinweis des Verlags Der Verlag bleibt in Hinblick auf geografische Zuordnungen und Gebietsbezeichnungen in veröffentlichten Karten und Institutsadressen neutral.

Open Access Dieser Artikel wird unter der Creative Commons Namensnennung 4.0 International Lizenz veröffentlicht, welche die Nutzung, Vervielfältigung, Bearbeitung, Verbreitung und Wiedergabe in jeglichem Medium und Format erlaubt, sofern Sie den/die ursprünglichen Autor(en) und die Quelle ordnungsgemäß nennen, einen Link zur Creative Commons Lizenz beifügen und angeben, ob Änderungen vorgenommen wurden. Die in diesem Artike enthaltenen Bilder und sonstiges Drittmaterial unterliegen ebenfalls der genannten Creative Commons Lizenz, sofern sich aus der Abbildungslegende nichts anderes ergibt. Sofern das betreffende Material nicht unter der genannten Creative Commons Lizenz steht und die betreffende Handlung nicht nach gesetzlichen Vorschriften erlaubt ist, ist für die oben aufgeführten Weiterverwendungen des Materials die Einwilligung des jeweiligen Rechteinhabers einzuholen. Weitere Details zur Lizenz entnehmen Sie bitte der Lizenzinformation auf http://creativecommons.org/licenses/by/4.0/deed.de.

\section{Literatur}

1. Arens, M., Worrell, E., Eichhammer, W., Hasanbeigi, A., Zhang, Q. (2017): Pathways to a low-carbon iron and steel industry in the medium-term - the case of Germany. J. Clean. Prod., 163, 84-98. https://doi.org/10.1016/j.jclepro.2015.12.097.

2. Atabay, D. (2017): An open-source model for optimal design and operation of industrial energy systems. Energy, 121, 803-821. https://doi.org/10.1016/j.energy.2017.01. 030

3. Bhaskar, A., Assadi, M., Nikpey Somehsaraei, H. (2020): Decarbonization of the iron and steel industry with direct reduction of iron ore with Green hydrogen. Energies, 13 , 758. https://doi.org/10.3390/en13030758.

\section{Autoren}

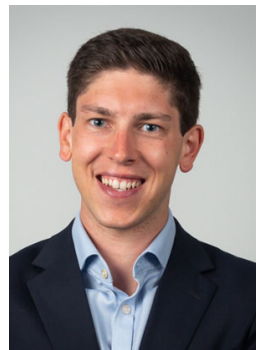

\section{Johannes Dock}

absolvierte an der Montanuniversität Leoben das Bachelorstudium „Petroleum Engineering" und erweiterte anschließend sein Fachgebiet durch den Wechsel in die Studienrichtung "Industrielle Energietechnik". 2017 verfasste er seine Masterarbeit am Lehrstuhl für Thermoprozesstechnik und beendete im Juni 2018 sein Masterstudium mit Auszeichnung Seit 2018 ist Johannes Dock als wissenschaftlicher Mitarbeiter am Lehrstuhl für Energieverbundtechnik tätig und unter anderem für die Abwicklung des NEFI-Projektes OxySteel verantwortlich.
4. Blesl, M., Kessler, A. (2013): Energieeffizienz in der Industrie. Berlin: Springer.

5. Çamdalı̈̈, T. M. (2002): Modelling of electric energy consumption in the AC electric arc furnace. Int. J. Energy Res., 26, 935-947. https://doi.org/10.1002/er.829.

6. Camdalı̈̈, T. M. (2003): Energy and exergy analysis of a ladle furnace. Can. Metall. Q., 42, 439-446. https://doi.org/10.1179/cmq.2003.42.4.439.

7. Chen, C., Liu, Y., Kumar, M., Qin, J. (2018): Energy consumption modelling using deep learning technique - a case study of EAF. Proc. CIRP, 72, 1063-1068. https://doi. org/10.1016/j.procir.2018.03.095.

8. Gajic, D., Savic-Gajic, I., Savic, I., Georgieva, O., Di Gennaro, S. (2016): Modelling of electrical energy consumption in an electric arc furnace using artificial neural networks. Energy, 108, 132-139. https://doi.org/10.1016/j.energy.2015.07.068.

9. Kirschen, M., Badr, K., Pfeifer, H. (2011): Influence of direct reduced iron on the energy balance of the electric arc furnace in steel industry. Energy, 36, 6146-6155. https:// doi.org/10.1016/j.energy.2011.07.050

10. Kovačič, M., Stopar, K., Vertnik, R., Šarler, B. (2019): Comprehensive electric arc furnace electric energy consumption modeling: a pilot study. Energies, 12, 2142. https:// doi.org/10.3390/en12112142.

11. Matino, I., Colla, V., Baragiola, S. (2017): Electric energy consumption and environmental impact in unconventional EAF steelmaking scenarios. Energy Proc., 105, 3636-3641. https://doi.org/10.1016/j.egypro.2017.03.839.

12. McKenna, E., Krawczynski, M., Thomson, M. (2015): Four-state domestic building occupancy model for energy demand simulations. Energy Build., 96, 30-39. https://doi. org/10.1016/j.enbuild.2015.03.013.

13. Remus, R. (2013): Best available techniques (BAT) reference document for iron and steel production: industrial emissions directive 2010/75/EU (integrated pollution prevention and control). Scientific and technical research series (Vol. 25521). Union, Luxembourg: Publications Office of the European.

14. Sasiain, A. Rechberger, K., Spanlang, A., Kofler, I., Wolfmeir, H., Harris, C., Bürgler, T. (2020): Green hydrogen as decarbonization element for the steel industry. Berg Huettenmaenn Monatsh, 165, 232-236. https://doi.org/10.1007/s00501-020-00968-1.

15. Shamshad, A., Bawadi, M., Wanhussin, W., Majid, T., Sanusi, S. (2005): First and second order Markov chain models for synthetic generation of wind speed time series. Energy, 30, 693-708. https://doi.org/10.1016/j.energy.2004.05.026.

16. Statistics Austria (2020): Energy balances Austria 1970-2019, Wien.

17. Stewart, W. (2009): Probability, Markov chains, queues, and simulation: the mathematical basis of performance modeling. Princeton (N.J.), Oxford: Princeton University Press.

18. Umweltbundesamt (2019): Austria's national inventory report 2019: submission under the United Nations framework convention on climate change and under the Kyoto protocol. Reports, vol. 0677, Wien.

19. Wang, Y., Infield, D. (2018): Markov chain Monte Carlo simulation of electric vehicle use for network integration studies. Int. J. Electr. Power Energy Syst., 99, 85-94. https://doi.org/10.1016/j.ijepes.2018.01.008.

20. Zarandi, M. F., Ahmadpour, P. (2009): Fuzzy agent-based expert system for steel making process. Expert Syst. Appl., 36, 9539-9547. https://doi.org/10.1016/j.eswa. 2008.10.084.

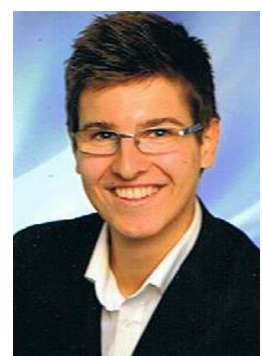

Daniel Janz

absolvierte das Studium Industrielle Umweltschutz- und Verfahrenstechnik an der Montanuniversität Leoben mit Spezialisierung in der Anlagentechnik und schloss dieses mit Auszeichnung ab. Seit Januar 2018 ist er als Prozesstechnologe bei der Breitenfeld Edelstahl AG, wo er sich mit Prozessoptimierung, Energieeffizienz, der Leitung von F\&E-Projekten sowie der Entwicklung von Software-Tools zur Datenanalyse beschäftigt.

Die Breitenfeld Edelstahl AG betreibt an ihrem Standort in St. Barbara-Mitterdorf im Mürztal den größten Elektrolichtbogenofen Österreichs. 


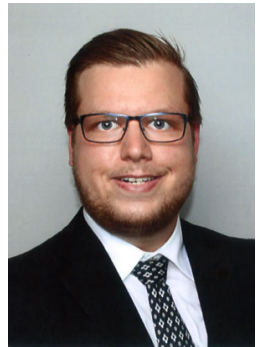

\section{Jakob Weiss}

absolvierte an der Montanuniversität Leoben sowohl das Bachelor- als auch das Masterstudium "Industrielle Energietechnik". Er verfasste seine Masterarbeit am Lehrstuhl für Thermoprozesstechnik und beendete sein Masterstudium im November 2020 mit Auszeichnung. Davor war er im Rahmen seiner Projektarbeit am Lehrstuhl für Energieverbundtechnik im NEFI-Projekt OxySteel involviert. Inzwischen leitet er die Planungsabteilung des PVGroßhändlers Suntastic.Solar.

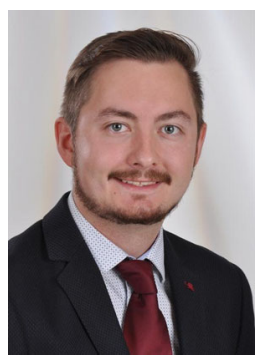

\section{Aaron Marschnig}

Nach Absolvierung des Bachelorstudiums "Industrielle Energietechnik" an der Montanuniversität Leoben im Jahr 2019 führte Aaron Marschnig das Masterstudium im selben Fachgebiet fort. Im Jahr 2020 verfasste er seine Masterarbeit im Rahmen des NEFIProjektes OxySteel am Lehrstuhl für Energieverbundtechnik und schloss sein Masterstudium im März 2021 ab. Seit Februar 2021 ist Aaron Marschnig als technischer Projektmanager bei der ElstaMosdorfer GmbH für die Weiterentwicklung des neuen Geschäftszweiges „Trafostationen" zuständig.

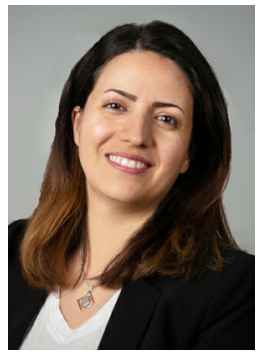

\section{Maedeh Rahnama Mobarakeh}

hat ihr Bachelor- und Masterstudium in "Maschinenbau" an iranischen Universitäten abgeschlossen und arbeitet seit 2018 als wissenschaftliche Mitarbeiterin am Lehrstuhl für Energieverbundtechnik an der Montanuniversität Leoben. Sie ist verantwortlich für das Thema Dekarbonisierung von Industriesektoren im Rahmen des NEFI_Lab-Projekts.

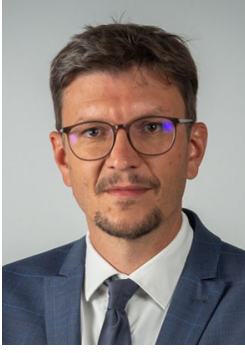

Thomas Kienberger

Seit 2014 ist Prof. Kienberger als Leiter des neu errichteten Lehrstuhls für Energieverbundtechnik an der Montanuniversität Leoben tätig. Dabei beschäftigt er sich in Forschung und Lehre schwerpunktmäßig mit Fragestellungen, die darauf abzielen mittels interdisziplinären, systemischen Ansätzen die Effizienz und Flexibilität von öffentlichen Energiesystemen sowie von industriellen Energiesystemen zu optimieren.

Prof. Kienberger absolvierte das Studium „Elektro-Energietechnik" an der TU Graz und schloss dieses mit Auszeichnung ab. Zwischen 2006 und 2007 arbeitete er als Entwicklungsingenieur bei der Firma Siemens AG in Erlangen/Deutschland. Von 2007 bis 2010 war er als wissenschaftlicher Assistent am Institut für Wärmetechnik an der TU Graz beschäftigt und leitete dabei unter anderem die Arbeitsgruppe "substitute natural gas". Von 2011 bis 2014 war er als Leiter R\&D und Prokurist beim Start-up agnion Highterm-Research tätig und fungierte gleichzeitig als Lehrbeauftragter an der TU-Graz. Neben seinen Aktivitäten in der Wirtschaft konnte Prof. Kienberger zahlreiche Forschungsprojekte initiieren und mehr als 50 Konferenz- und Journalbeträge veröffentlichen. 